\title{
A Study on Marketing Pattern of Onion in Nashik District of Maharashtra, India
}

\author{
Kumud Shukla ${ }^{1}$, Ghanshyam Kumar Pandey ${ }^{2 *}$, M. Vinaya Kumari ${ }^{1}$, \\ Avinash Vanam ${ }^{4}$ and Nahar Singh ${ }^{3}$
}

${ }^{1}$ Department of Agricultural Economics and Agri-business Management, ${ }^{2}$ Department of Plant Pathology, Faculty of Agriculture, ${ }^{3}$ Department of Agricultural Economics and Agri-

business Management, Sam Higginbottom University of Agriculture, Technology and Sciences, Allahabad, Uttar Pradesh, India

${ }^{4}$ Governments of India, Ministry of Agriculture \& Farmers Welfare, New Delhi-110003

*Corresponding author

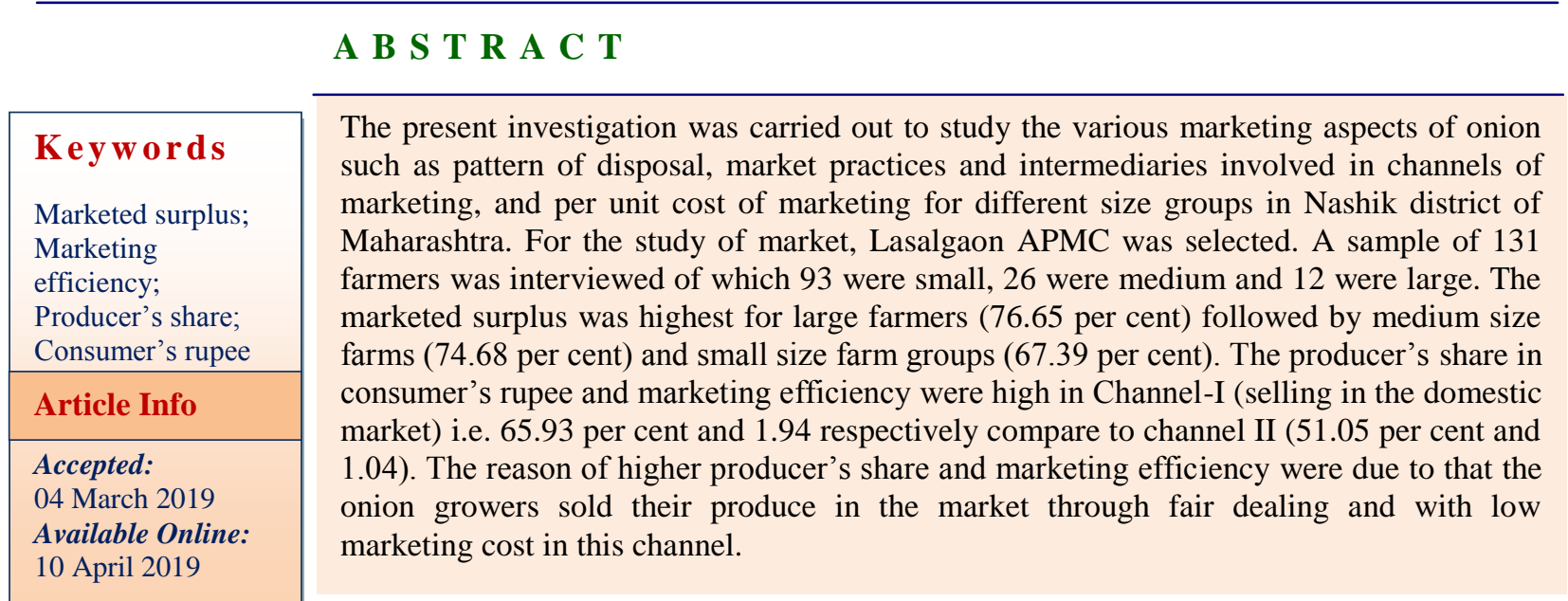

\section{Introduction}

Onion (Allium cepa) has an extensive culinary, dietary, therapeutic, trading, income and employment generation value. Onion is commodity of mass consumption and is grown almost all over the country mainly by small and marginal farmers as this is labour intensive crop. India ranks second after China having 1305.64 thousand ha. area and
22427.43 thousand metric tones production (Source: NHRDF, 2016-17). The three main seasons of kharif (monsoon), late kharif and rabi (winter) contribute $15 \%, 20 \%$ and $65 \%$, respectively to the total onion production (Source: NHB, 2016-17).

Maharashtra ranks first state in onion production with share of 30.03 per cent therefore it is called as 'onion basket of India' 
(Source: www.apeda.com). In Maharashtra, area, production and productivity of onion in year 20016-17 was 481.05 thousand ha, 6734.74 thousand metric tones and 14 ton/ha. Respectively (Source: NHRDF). The district of Nashik in Maharashtra accounts for the largest share in the production of onions in India. Nashik onion is not only consumed in the farthest corners of India, it is also exported to many countries. Onion is a major item of agricultural exports, earning valuable foreign exchange to the country. Onion poses more problems as compared to other agricultural commodities due to seasonality and high demand. It adversely affects the economy of the farmers that there is need to call from Government and policy makers to pay attention on effective planning. Presently development of marketing infrastructure and price support of onion are main concern of government to solve the problems of onion growers. However, more intensified efforts are needed to identify the specific problems related to onion marketing.

The present investigation was undertaken with the objectives to estimate the marketable surplus and marketed surplus in the study area in various size groups of farmers and to calculate the price spread and marketing efficiency of onion in Nashik district of Maharashtra.

\section{Materials and Methods}

The study was conducted in Nashik district of Maharashtra State during the year 2016-17. Nashik district was selected purposively as having remarkable onion production in Maharashtra. Out of 15 blocks of Nashik district, Niphad and Yeola blocks were selected for the study because leading onion producing blocks and higher access to markets. In selected blocks, seven villages were selected from Niphad based on highest area under onion crop; similarly six villages from Yeola were selected. From each village, ten per cent farmers were selected randomly. Hence, the study covered 13 villages from 2 blocks of Nashik district to form a sample of 131 respondents. A pre-tested structured interview schedule was used to collect the data from the respondents by personal interview method. In total 131 farmers were interviewed in the study of which 93 were marginal, 26 were small and 12 were large. For the study of market, Lasalgaon APMC was selected which is Asia's biggest onion market. The data pertaining to onion prices were obtained from onion retail stores in Niphad and Yeola blocks. 2 per cent functionaries were selected for collection of data regarding marketing and price spread of onion in different channels of marketing. Altogether total in numbers market functionaries were viz.19, 5 wholesalers, 5 exporters and 9 retailers were chosen for the study.

Two marketing channels were observed in the study area as follows:

Channel-I $=$ Producer $\rightarrow$ Commission agent/Wholesaler $\rightarrow$ Retailer $\rightarrow$ Consumer

Channel-II $=$ Producer $\rightarrow$ Commission agent/Wholesaler $\rightarrow$ Exporter $\rightarrow$ International Buyer

\section{Analytical tools}

\section{Marketable surplus}

In this study the term marketable surplus was used to denote the quantity which was a real of the surplus under varying conditions after the consumption and other requirements of the farmer were met? It was computed by the formula:

Marketable surplus $(\mathrm{MS})=\mathrm{P}-\mathrm{C}$ 
Where $\mathrm{P}=$ Gross production, $\mathrm{C}=$ Total requirement

\section{Marketed surplus}

In this study the term marketed surplus was used to denote the actual quantum of sales by the production irrespective of requirements.

Relation between marketed surplus and marketable surplus: Marketed surplus may be less than, equal to or greater than marketable surplus. Mostly in case of small and medium farmers marketed surplus is higher than marketable surplus.

\section{Marketing channel}

The chain of intermediaries through which the various farm commodities pass between producers and consumers is called a marketing channel. Major marketing channels in the transportation of onion from farmer to the ultimate consumer were identified. The volumes of transaction through each channel were estimated to calculate the effectiveness of each channel.

\section{Producer's share in consumer's rupee}

It is price received by the farmer to the retail price expressed as percentage. If $\mathrm{pr}$ is the retail price and $\mathrm{Pf}$ is the price received by the farmer then the producer's share in consumer's rupee Ps may be expressed as follows.

$\mathrm{Ps}=(\mathrm{Pf} / \mathrm{Pr}) \times 100$

\section{Price spread}

Price spread is the difference between the price paid by the consumer and the price received by the producer. It mainly consists of marketing cost and marketing margin. The price spread analysis was carried out as follows:
Producer's share in consumer's rupee $=$ Producer's price/consumer's price x 100

\section{Marketing margin of the middlemen}

This is the difference between the total payments (cost + purchase price) and receipts (sale price) of the middleman, the ith agency. Percentage margin of the ith middlemen $($ pmi $)=$ pri $-($ ppi $+\mathrm{cmi}) /$ pri $\times 100$

Where Pri= Total value receipts per unit (sale price), $\mathrm{ppi}=$ Purchase value of goods per unit, $\mathrm{cmi}=$ Cost incurred in marketing per unit

\section{Total cost of marketing}

The total cost incurred on the marketing either in cash or in kind by the producer seller and other various intermediaries involved in the sale and the purchase of the commodity till the commodity reach the consumers may be computed as follows:

$\mathrm{C}=\mathrm{Cf}+\mathrm{Cm} 1+\mathrm{Cm} 2+$ $+\mathrm{Cmn}$ where $\mathrm{C}=$ Total cost of the marketing of the commodity, $\mathrm{Cf}=$ Total cost paid by the producer from the time of the produce leaves the farm till he sells it, $\mathrm{Cmi}=$ Cost incurred by the ith middle man in the process of buying and selling the product

\section{Marketing efficiency}

Marketing efficiency is the ratio of the market output to market input. An increase in this ratio represents improved efficiency and decrease denotes reduced efficiency. It is effectiveness or competence with which a market structure performs its designed function. Marketing efficiency is represented as follows:

$\mathrm{ME}=\mathrm{V} / \mathrm{I}-1$ (Shepherd's formula) where $\mathrm{ME}=$ Index of marketing efficiency, $\mathrm{V}=$ Value of goods sold, I= Total marketing cost 


\section{Results and Discussion}

\section{Disposal pattern of onion in sample farms and marketing channels (Table 1)}

Total production of onion in quintals was highest in large size farms (206.97 qts) as compared medium (127.48 qtls) and was lowest in small size farms (43.43qtls). The quantity retained for onion growers was mostly for home consumption, some of the quantity was used as kind payment to labours as wages, some of the quantity used as gift for religious purpose and finally they retain some quantity for next year. The highest per cent of the produce was retained by small size farms (32.60 per cent) followed by medium size farms (25.31 per cent) and large size farms (23.34 per cent) respectively. This also indicated that highest percentage marketable surplus was found by large size farms 76.65 per cent followed by 74.68 per cent in medium size farms and 67.39 per cent in small size of farm groups. This makes the sample average for marketable surplus of 72.91 per cent of the total production. The same result was generated by Baba et al., (2010), Gaurav (2011) and Sashimatsung (2015). It could be seen from the table that actual marketed surplus was highest in large size farms (158.64 qtls) followed by medium and small size of farm groups ( 95.20 and 29.27/qtls) respectively. The table revealed that disposal pattern of actual marketable surplus of Onion in two different marketing channels i.e. channel I and channel II was most prevalent adopted by the growers in the study area, as the highest percentage of the produce was transacted trough channel I i.e. 82.89 per cent of growers and 22.48 per cent through channel II.

Price spread of onion (one quintal) in different channels (Table $2 \& 3$ )

In channel-I (Producer $\rightarrow$ Commission agent/Wholesaler $\rightarrow$ Retailer $\rightarrow$ Consumer $)$ average marketing cost when producers sold their produce to commission agents/wholesalers in the market was Rs. 75.26/qtl. Sale price of the producer to commission agents/ wholesalers was Rs. 646.91/qtl in different farm size groups. This is conformity with the result of Jagtap (2014). Average marketing cost incurred by wholesaler/ commission agent was Rs. 173.16 and margin of wholesaler/ commission agent was Rs. 107.24. However, an expense incurred by the retailer was Rs 161.14, margin of retailer was Rs 102.83 and the consumer's purchasing price was Rs 981.21.

In this channel, marketing cost of the producer, commission agents/wholesalers and retailers was 7.67 per cent, 17.65 per cent and 16.26 per cent of consumers paid price respectively. The commission agent/ wholesalers margin was estimated to be 10.93 per cent and the retailer's margin was 10.48 per cent of the consumer paid price. Producer share in consumer price was highest on large size farms (67.06 per cent) as compared to medium and small size of farm groups (65.60 per cent and 65.11 per cent) on respectively. Price spread was highest in small size farms which constituted to Rs. 340.51/qtl of consumer paid price (Table 2). The result showed low producer's share in consumer price. This is due to onion growers did not have any control over the market due to the absence of coordination and integration among themselves. All the expenses in the marketing process are incurred by the producers practically the retailers or buyer charges paid to mandi are also charged from the producer (Barakade et al., 2011).

The channel-II (Producer $\rightarrow$ Commission agent/Wholesaler $\rightarrow$ Exporter $\rightarrow$ International Buyer) was found prevalent in study area due to export variety of onion. Average marketing cost incurred by producers was Rs.124.10/qtl and sale price was Rs.773.33/qtl in different farms size group. Average marketing cost 
incurred by wholesaler/ commission agent was Rs. 202.67and margin of wholesaler/ commission agent was Rs. 82.73. However, an expense incurred by the exporter was Rs 538.48, margin of exporters was Rs 106.35 and the consumer's purchasing price was Rs 1514.48. In this channel, marketing cost of the producer, commission agents/wholesalers and exporter was 8.19 per cent, 13.37 per cent and 35.56 per cent of buyers paid price respectively. The commission agent/ wholesalers margin was estimated to be 5.46 per cent and the exporter's margin was 7.03 per cent of the buyer paid price. Producer share in buyer price was highest on large size farms (52.33 per cent) as compared to medium and small size of farm groups (50.90 per cent and 49.93 per cent) on respectively. Price spread was highest in small size farms which constituted to Rs.747.42/qtl of consumer paid price (Table 3 ).

\section{Indices of marketing efficiency in different channel (Table 4)}

Marketing efficiency is an effective agent of change and an important means for raising the income level of the farmers. As data indicated that the marketing efficiency of onion was found to be the higher i.e. 1.94 per cent in case of channel I compare to channel II (1.04 per cent).

Table.1 Utilization of produce in sample farms of three sizes and in different channels

\begin{tabular}{|c|l|c|c|c|c|}
\hline \multirow{2}{*}{$\begin{array}{c}\text { Sl. } \\
\text { No }\end{array}$} & \multicolumn{1}{|c|}{ Particulars } & \multicolumn{3}{|c|}{ Size of farm groups } & Sample \\
& & Small & Medium & Large & \\
\hline $\mathbf{1}$ & Total production of onion in quintals Per & 43.43 & 127.48 & 206.97 & 125.96 \\
& farm level & $(100)$ & $(100)$ & $(100)$ & $(100)$ \\
\hline $\mathbf{2}$ & Retained onion (in quintals) & & & & \\
\hline $\mathbf{I}$ & Home consumption & 3.77 & 6.50 & 8.20 & 6.16 \\
& & $(8.69)$ & $(5.1)$ & $(3.96)$ & $(5.92)$ \\
\hline Ii & Kind payment as wages & 5.66 & 10.85 & 16.04 & 10.85 \\
& & $(13.04)$ & $(8.51)$ & $(7.75)$ & $(9.77)$ \\
\hline Iii & Relatives and religious person & 3.77 & 10.85 & 20.84 & 11.82 \\
& & $(8.69)$ & $(8.51)$ & $(10.07)$ & $(9.09)$ \\
\hline Iv & Retain for next years & 0.94 & 4.07 & 3.21 & 2.74 \\
& & $(2.17)$ & $(3.19)$ & $(1.55)$ & $(2.30)$ \\
\hline $\mathbf{4}$ & Total retention for onion & 14.16 & 32.27 & 48.30 & 31.58 \\
& & $(32.60)$ & $(25.31)$ & $(23.34)$ & $(27.08)$ \\
\hline $\mathbf{5}$ & Marketable surplus & 29.27 & 95.20 & 158.64 & 94.37 \\
& & $(67.39)$ & $(74.68)$ & $(76.65)$ & $(72.91)$ \\
\hline $\mathbf{7}$ & Quantity purchased from other farm & 6 & 12 & 20 & 11 \\
\hline $\mathbf{8}$ & Marketed surplus & 35.27 & 107.20 & 178.64 & 105.37 \\
& & $(100.00)$ & $(100.00)$ & $(100.00)$ & $(100.00)$ \\
\hline $\mathbf{9}$ & Disposal of actual marketed surplus of onion & & & & \\
\hline & in different marketing channels & & & & \\
\hline I & Producer $\rightarrow$ Commission agent/Wholesaler & 28.98 & 83.56 & 140.04 & 82.89 \\
& $\rightarrow$ Retailer $\rightarrow$ Consumer & $(82.17)$ & $(77.95)$ & $(78.39)$ & $(78.67)$ \\
\hline II & Producer $\rightarrow$ Commission agent/Wholesaler & 6.29 & 23.64 & 38.60 & 22.48 \\
& $\rightarrow$ Exporter & $(17.83)$ & $(22.05)$ & $(21.61)$ & $(21.33)$ \\
\hline
\end{tabular}


Table.2 Price spread of onion in different size of farm groups for channel I

\begin{tabular}{|c|c|c|c|c|c|}
\hline \multirow[t]{2}{*}{ SI. No } & \multirow[t]{2}{*}{ Particulars } & \multicolumn{3}{|c|}{ Size of farm groups } & \multirow{2}{*}{$\begin{array}{l}\text { Sample } \\
\text { Average }\end{array}$} \\
\hline & & Small & Medium & Large & \\
\hline 1 & Producer sale price to Commission agent & 635.53 & 643.78 & 661.43 & 646.91 \\
\hline 2 & Cost incurred by the producer & & & & \\
\hline I & Packing cost & $\begin{array}{c}5.22 \\
(0.54)\end{array}$ & $\begin{array}{c}5.92 \\
(0.60)\end{array}$ & $\begin{array}{c}6.48 \\
(0.66)\end{array}$ & $\begin{array}{c}5.88 \\
(0.60)\end{array}$ \\
\hline Ii & Packing material cost & $\begin{array}{c}7.04 \\
(0.72)\end{array}$ & $\begin{array}{c}6.45 \\
(0.66)\end{array}$ & $\begin{array}{c}7.12 \\
(0.72)\end{array}$ & $\begin{array}{c}6.87 \\
(0.70)\end{array}$ \\
\hline iii & Transportation cost & $\begin{array}{l}10.5 \\
(1.07)\end{array}$ & $\begin{array}{l}10.57 \\
(1.08)\end{array}$ & $\begin{array}{c}8.28 \\
(0.84)\end{array}$ & $\begin{array}{c}9.78 \\
(0.99)\end{array}$ \\
\hline Iv & Market cost & $\begin{array}{c}7.35 \\
(0.75)\end{array}$ & $\begin{array}{c}7.08 \\
(0.72)\end{array}$ & $\begin{array}{c}7.65 \\
(0.77)\end{array}$ & $\begin{array}{c}7.36 \\
(0.75)\end{array}$ \\
\hline $\mathbf{V}$ & Labour cost & $\begin{array}{l}13.02 \\
(1.33)\end{array}$ & $\begin{array}{l}11.84 \\
(1.21)\end{array}$ & $\begin{array}{c}8.60 \\
(0.87)\end{array}$ & $\begin{array}{l}11.15 \\
(1.14)\end{array}$ \\
\hline vi & Loading and unloading charges & $\begin{array}{c}6.51 \\
(0.67)\end{array}$ & $\begin{array}{c}7.82 \\
(0.79)\end{array}$ & $\begin{array}{c}8.60 \\
(0.87)\end{array}$ & $\begin{array}{c}7.64 \\
(0.78)\end{array}$ \\
\hline vii & Weighing charges & $\begin{array}{c}6.20 \\
(0.63)\end{array}$ & $\begin{array}{c}6.76 \\
(0.69)\end{array}$ & $\begin{array}{c}7.33 \\
(0.74)\end{array}$ & $\begin{array}{c}6.76 \\
(0.69)\end{array}$ \\
\hline viii & Miscellaneous charges & $\begin{array}{l}18.38 \\
(1.88)\end{array}$ & $\begin{array}{l}19.02 \\
(1.94)\end{array}$ & $\begin{array}{l}21.99 \\
(2.23)\end{array}$ & $\begin{array}{l}19.80 \\
(2.01)\end{array}$ \\
\hline 3 & Total cost (i-viii) & $\begin{array}{l}74.26 \\
(7.61)\end{array}$ & $\begin{array}{l}75.46 \\
(7.69)\end{array}$ & $\begin{array}{l}76.05 \\
(7.71)\end{array}$ & $\begin{array}{l}75.26 \\
(7.67)\end{array}$ \\
\hline 4 & Net price received by producer & $\begin{array}{l}561.27 \\
(57.50)\end{array}$ & $\begin{array}{l}568.32 \\
(57.91)\end{array}$ & $\begin{array}{l}585.38 \\
(59.35)\end{array}$ & $\begin{array}{l}571.66 \\
(58.26)\end{array}$ \\
\hline 5 & $\begin{array}{l}\text { Sale price of producer to } \\
\text { Commission agent/ Wholesaler }\end{array}$ & $\begin{array}{l}635.53 \\
(65.11)\end{array}$ & $\begin{array}{l}643.78 \\
(65.60)\end{array}$ & $\begin{array}{l}661.43 \\
(67.06)\end{array}$ & $\begin{array}{l}646.91 \\
(65.93)\end{array}$ \\
\hline 6 & $\begin{array}{l}\text { Cost incurred by the } \\
\text { Commission agent/ Wholesaler }\end{array}$ & & & & \\
\hline I & Loading and unloading charges & $\begin{array}{l}9.45 \\
(0.97)\end{array}$ & $\begin{array}{c}9.72 \\
(0.99)\end{array}$ & $\begin{array}{r}10.62 \\
(1.07)\end{array}$ & $\begin{array}{c}9.93 \\
(1.01)\end{array}$ \\
\hline Ii & Grading & $\begin{array}{l}11.24 \\
(1.15)\end{array}$ & $\begin{array}{l}11.52 \\
(1.17)\end{array}$ & $\begin{array}{r}11.79 \\
(1.19)\end{array}$ & $\begin{array}{l}11.52 \\
(1.17)\end{array}$ \\
\hline iii & Packing & $\begin{array}{l}9.14 \\
(0.94)\end{array}$ & $\begin{array}{c}9.41 \\
(0.96)\end{array}$ & $\begin{array}{c}9.77 \\
(0.99)\end{array}$ & $\begin{array}{c}9.44 \\
(0.96)\end{array}$ \\
\hline Iv & Market fee & $\begin{array}{l}12.39 \\
(1.27)\end{array}$ & $\begin{array}{l}12.68 \\
(1.29)\end{array}$ & $\begin{array}{l}13.06 \\
(1.32)\end{array}$ & $\begin{array}{l}12.71 \\
(1.29)\end{array}$ \\
\hline $\mathbf{V}$ & $\begin{array}{l}\text { Commission of } \\
\text { Commission agent/ Wholesaler }\end{array}$ & $\begin{array}{l}9.45 \\
(0.97)\end{array}$ & $\begin{array}{c}9.72 \\
(0.99)\end{array}$ & $\begin{array}{r}10.09 \\
(1.02)\end{array}$ & $\begin{array}{c}9.75 \\
(0.99)\end{array}$ \\
\hline vi & Losses \& miscellaneous charges & $\begin{array}{l}13.34 \\
(1.37)\end{array}$ & $\begin{array}{l}13.00 \\
(1.32)\end{array}$ & $\begin{array}{r}11.37 \\
(1.15)\end{array}$ & $\begin{array}{l}12.57 \\
(1.28)\end{array}$ \\
\hline vii & Commission agent/ Wholesaler Margin & $\begin{array}{l}110.00 \\
(11.27)\end{array}$ & $\begin{array}{l}111.08 \\
(11.32)\end{array}$ & $\begin{array}{l}100.64 \\
(10.20)\end{array}$ & $\begin{array}{l}107.24 \\
(10.93)\end{array}$ \\
\hline 7 & Total cost (i-vii) & $\begin{array}{l}175.01 \\
(17.93)\end{array}$ & $\begin{array}{l}177.13 \\
(18.05)\end{array}$ & $\begin{array}{l}167.34 \\
(16.97)\end{array}$ & $\begin{array}{l}173.16 \\
(17.65)\end{array}$ \\
\hline
\end{tabular}




\begin{tabular}{|c|l|c|c|c|c|}
\hline $\mathbf{8}$ & Sale price of /Commission agent & 810.54 & 820.91 & 828.77 & 820.07 \\
& wholesalers to Retailers & $(83.04)$ & $(83.65)$ & $(84.03)$ & $(83.57)$ \\
\hline $\mathbf{9}$ & Cost incurred by the retailers & & & & \\
\hline $\mathbf{I}$ & Weighing charges & 10.92 & 10.57 & 11.26 & 10.92 \\
& & $(1.12)$ & $(1.08)$ & $(1.14)$ & $(1.11)$ \\
\hline $\mathbf{I i}$ & Loading and unloading charges & 9.45 & 10.36 & 10.62 & 10.14 \\
& & $(0.97)$ & $(1.05)$ & $(1.08)$ & $(1.03)$ \\
\hline $\mathbf{i i i}$ & Town charges & 6.51 & 7.93 & 7.97 & 7.47 \\
& & $(0.67)$ & $(0.81)$ & $(0.81)$ & $(0.76)$ \\
\hline $\mathbf{I v}$ & Carriage up to shop & 15.12 & 18.07 & 18.06 & 17.08 \\
& & $(1.55)$ & $(1.84)$ & $(1.83)$ & $(1.74)$ \\
\hline $\mathbf{V}$ & Miscellaneous charges & 19.11 & 9.93 & 9.03 & 12.69 \\
& & $(1.96)$ & $(1.01)$ & $(0.91)$ & $(1.29)$ \\
\hline $\mathbf{v i}$ & Retailers margin & 104.37 & 103.57 & 100.56 & 102.83 \\
& & $(10.69)$ & $(10.55)$ & $(10.19)$ & $(10.48)$ \\
\hline $\mathbf{1 0}$ & Total cost (i-vi) & 165.50 & 160.43 & 157.49 & 161.14 \\
& & $(16.96)$ & $(16.34)$ & $(15.96)$ & $(16.42)$ \\
\hline $\mathbf{1 1}$ & Sale price of retailers to consumers & 976.04 & 981.34 & 986.26 & 981.21 \\
& & $(100)$ & $(100)$ & $(100)$ & $(100)$ \\
\hline $\mathbf{1 2}$ & Price spread & 340.51 & 337.56 & 324.83 & 334.30 \\
& & $(34.87)$ & $(34.40)$ & $(32.94)$ & $(34.07)$ \\
\hline $\mathbf{1 3}$ & Producer's share in consumer's rupee (\%) & 65.11 & 65.60 & 67.06 & 65.93 \\
\hline
\end{tabular}

Table.3 Price spread of onion in different size of farm groups for channel II

\begin{tabular}{|c|c|c|c|c|c|}
\hline \multirow{2}{*}{$\begin{array}{l}\text { Sl. } \\
\text { No }\end{array}$} & \multirow[t]{2}{*}{ Particulars } & \multicolumn{3}{|c|}{ Size of farm groups } & \multirow{2}{*}{$\begin{array}{l}\text { Sample } \\
\text { Average }\end{array}$} \\
\hline & & Small & Medium & Large & \\
\hline 1 & Producer sale price to commission agent & 745.32 & 773.56 & 801.12 & 773.33 \\
\hline 2 & Cost incurred by the producer & & & & \\
\hline $\mathbf{I}$ & Packing cost & $\begin{array}{c}10.98 \\
(0.73)\end{array}$ & $\begin{array}{l}12.32 \\
(0.81)\end{array}$ & $\begin{array}{r}13.60 \\
(0.88)\end{array}$ & $\begin{array}{l}12.30 \\
(0.81)\end{array}$ \\
\hline Ii & Packing material cost & $\begin{array}{c}15.99 \\
(1.07)\end{array}$ & $\begin{array}{l}13.63 \\
(0.89)\end{array}$ & $\begin{array}{r}14.76 \\
(0.96)\end{array}$ & $\begin{array}{l}14.79 \\
(0.97)\end{array}$ \\
\hline iii & Transportation cost & $\begin{array}{c}17.12 \\
(1.14)\end{array}$ & $\begin{array}{l}16.09 \\
(1.06)\end{array}$ & $\begin{array}{c}17.25 \\
(1.13)\end{array}$ & $\begin{array}{l}16.82 \\
(1.11)\end{array}$ \\
\hline Iv & Market cost & $\begin{array}{l}15.34 \\
(1.02)\end{array}$ & $\begin{array}{l}14.78 \\
(0.97)\end{array}$ & $\begin{array}{c}16.09 \\
(1.05)\end{array}$ & $\begin{array}{l}15.40 \\
(1.01)\end{array}$ \\
\hline $\mathbf{V}$ & Labour cost & $\begin{array}{c}18.41 \\
(1.23)\end{array}$ & $\begin{array}{l}16.75 \\
(1.10)\end{array}$ & $\begin{array}{c}17.91 \\
(1.17)\end{array}$ & $\begin{array}{l}17.69 \\
(1.17)\end{array}$ \\
\hline vi & Loading and unloading charges & $\begin{array}{r}13.56 \\
(0.91)\end{array}$ & $\begin{array}{l}17.41 \\
(1.14)\end{array}$ & $\begin{array}{c}17.91 \\
(1.17)\end{array}$ & $\begin{array}{l}16.29 \\
(1.07)\end{array}$ \\
\hline vii & Weighing charges & $\begin{array}{r}12.92 \\
(0.86) \\
\end{array}$ & $\begin{array}{l}14.29 \\
(0.94) \\
\end{array}$ & $\begin{array}{r}15.43 \\
(1.00) \\
\end{array}$ & $\begin{array}{c}14.21 \\
(0.93)\end{array}$ \\
\hline viii & Miscellaneous charges & $\begin{array}{c}17.76 \\
(1.19)\end{array}$ & $\begin{array}{l}17.24 \\
(1.13)\end{array}$ & $\begin{array}{r}14.76 \\
(0.96)\end{array}$ & $\begin{array}{l}16.59 \\
(1.09)\end{array}$ \\
\hline
\end{tabular}




\begin{tabular}{|c|c|c|c|c|c|}
\hline 3 & Total cost (i-viii) & $\begin{array}{l}122.08 \\
(8.18)\end{array}$ & $\begin{array}{l}122.51 \\
(8.06)\end{array}$ & $\begin{array}{l}127.72 \\
(8.34)\end{array}$ & $\begin{array}{c}124.10 \\
(8.19)\end{array}$ \\
\hline 4 & Net price received by producer & $\begin{array}{l}623.24 \\
(41.75)\end{array}$ & $\begin{array}{l}651.05 \\
(42.84)\end{array}$ & $\begin{array}{l}673.40 \\
(43.98)\end{array}$ & $\begin{array}{l}649.23 \\
(42.86)\end{array}$ \\
\hline 5 & $\begin{array}{l}\text { Sale price of producer to } \\
\text { Commission agent/ Wholesaler }\end{array}$ & $\begin{array}{l}745.32 \\
(49.93)\end{array}$ & $\begin{array}{l}773.56 \\
(50.90)\end{array}$ & $\begin{array}{l}801.12 \\
(52.33)\end{array}$ & $\begin{array}{l}773.33 \\
(51.05)\end{array}$ \\
\hline 6 & $\begin{array}{l}\text { Cost incurred by the Commission } \\
\text { agent/ Wholesaler }\end{array}$ & & & & \\
\hline I & Loading and unloading charges & $\begin{array}{l}22.76 \\
(1.52)\end{array}$ & $\begin{array}{r}18.39 \\
(1.21)\end{array}$ & $\begin{array}{l}16.59 \\
(1.08)\end{array}$ & $\begin{array}{l}19.25 \\
(1.27)\end{array}$ \\
\hline Ii & Grading & $\begin{array}{l}19.70 \\
(1.31)\end{array}$ & $\begin{array}{l}17.90 \\
(1.18)\end{array}$ & $\begin{array}{l}18.41 \\
(1.20)\end{array}$ & $\begin{array}{l}18.67 \\
(1.23)\end{array}$ \\
\hline iii & Packing & $\begin{array}{l}29.71 \\
(1.99)\end{array}$ & $\begin{array}{l}26.44 \\
(1.74)\end{array}$ & $\begin{array}{l}25.21 \\
(1.64)\end{array}$ & $\begin{array}{l}27.12 \\
(1.79)\end{array}$ \\
\hline Iv & Market fee & $\begin{array}{l}19.05 \\
(1.27)\end{array}$ & $\begin{array}{l}19.71 \\
(1.29)\end{array}$ & $\begin{array}{l}20.40 \\
(1.33)\end{array}$ & $\begin{array}{l}19.72 \\
(1.30)\end{array}$ \\
\hline $\mathbf{V}$ & $\begin{array}{l}\text { Commission of Commission agent/ } \\
\text { Wholesaler }\end{array}$ & $\begin{array}{l}14.53 \\
(0.97)\end{array}$ & $\begin{array}{l}15.11 \\
(0.99)\end{array}$ & $\begin{array}{l}15.76 \\
(1.02)\end{array}$ & $\begin{array}{l}15.13 \\
(0.99)\end{array}$ \\
\hline vi & Losses \& miscellaneous charges & $\begin{array}{l}20.51 \\
(1.37)\end{array}$ & $\begin{array}{l}20.20 \\
(1.33)\end{array}$ & $\begin{array}{l}19.41 \\
(1.27)\end{array}$ & $\begin{array}{l}20.04 \\
(1.32)\end{array}$ \\
\hline vii & Commission agent/ Wholesaler margin & $\begin{array}{l}80.88 \\
(5.42)\end{array}$ & $\begin{array}{l}86.12 \\
(5.66)\end{array}$ & $\begin{array}{c}81.2 \\
(5.30)\end{array}$ & $\begin{array}{l}82.73 \\
(5.46)\end{array}$ \\
\hline 7 & Total cost (i-vii) & $\begin{array}{l}207.16 \\
(13.88)\end{array}$ & $\begin{array}{l}203.87 \\
(13.41)\end{array}$ & $\begin{array}{l}196.98 \\
(12.87)\end{array}$ & $\begin{array}{l}202.67 \\
(13.37)\end{array}$ \\
\hline 8 & $\begin{array}{l}\text { Sale price of /Commission agent } \\
\text { wholesalers to exporter }\end{array}$ & $\begin{array}{l}952.48 \\
(63.81)\end{array}$ & $\begin{array}{l}977.43 \\
(64.32)\end{array}$ & $\begin{array}{l}998.10 \\
(65.19)\end{array}$ & $\begin{array}{l}976.00 \\
(64.44)\end{array}$ \\
\hline 9 & Cost incurred by the exporters & & & & \\
\hline $\mathbf{I}$ & Transportation charges & $\begin{array}{l}138.88 \\
(9.30)\end{array}$ & $\begin{array}{l}122.51 \\
(8.06)\end{array}$ & $\begin{array}{l}127.72 \\
(8.34)\end{array}$ & $\begin{array}{c}129.70 \\
(8.57)\end{array}$ \\
\hline Ii & Freight charges to port of shipment & $\begin{array}{c}101.73 \\
(6.81)\end{array}$ & $\begin{array}{c}105.10 \\
(6.92)\end{array}$ & $\begin{array}{l}107.82 \\
(7.04)\end{array}$ & $\begin{array}{c}104.88 \\
(6.92)\end{array}$ \\
\hline iii & Clearing and forwarding charges & $\begin{array}{l}50.38 \\
(3.37)\end{array}$ & $\begin{array}{l}54.36 \\
(3.57)\end{array}$ & $\begin{array}{l}56.40 \\
(3.68)\end{array}$ & $\begin{array}{l}53.71 \\
(3.54)\end{array}$ \\
\hline Iv & $\begin{array}{l}\text { Dock charges / wharf age/ terminal } \\
\text { handling charges etc. }\end{array}$ & $\begin{array}{l}100.12 \\
(6.71)\end{array}$ & $\begin{array}{l}103.62 \\
(6.81)\end{array}$ & $\begin{array}{l}105.49 \\
(6.89)\end{array}$ & $\begin{array}{l}103.08 \\
(6.80)\end{array}$ \\
\hline $\mathbf{v}$ & Commission & $\begin{array}{l}10.98 \\
(0.73)\end{array}$ & $\begin{array}{l}11.33 \\
(0.74)\end{array}$ & $\begin{array}{l}11.78 \\
(0.77)\end{array}$ & $\begin{array}{l}11.36 \\
(0.75)\end{array}$ \\
\hline vi & Miscellaneous charges & $\begin{array}{l}28.42 \\
(1.90)\end{array}$ & $\begin{array}{l}29.40 \\
(1.93)\end{array}$ & $\begin{array}{l}30.35 \\
(1.98)\end{array}$ & $\begin{array}{l}29.39 \\
(1.94)\end{array}$ \\
\hline vii & Exporters margin & $\begin{array}{l}109.75 \\
(7.35)\end{array}$ & $\begin{array}{l}115.96 \\
(7.63)\end{array}$ & $\begin{array}{l}93.34 \\
(6.07)\end{array}$ & $\begin{array}{l}106.35 \\
(7.03)\end{array}$ \\
\hline 10 & Total cost (i-vii) & $\begin{array}{l}540.26 \\
(36.19)\end{array}$ & $\begin{array}{l}542.28 \\
(35.68)\end{array}$ & $\begin{array}{l}532.90 \\
(34.81)\end{array}$ & $\begin{array}{l}538.48 \\
(35.56)\end{array}$ \\
\hline 11 & Sale price of exporters to buyers & 1492.74 & 1519.71 & 1531.00 & 1514.48 \\
\hline
\end{tabular}




\begin{tabular}{|c|l|c|c|c|c|}
\hline & & $(100)$ & $(100)$ & $(100)$ & $(100)$ \\
\hline 12 & Price spread & 747.42 & 746.15 & 729.88 & 741.15 \\
& & $(50.07)$ & $(49.09)$ & $(47.67)$ & $(48.94)$ \\
\hline 13 & Producer's share in buyer's rupee (\%) & 49.93 & 50.90 & 52.33 & 51.05 \\
\hline
\end{tabular}

Table.4 Indices of marketing efficiency in different channels

\begin{tabular}{|l|l|c|c|}
\hline \multirow{2}{*}{$\begin{array}{l}\text { Ni. } \\
\text { No. }\end{array}$} & Particular & \multicolumn{2}{|c|}{ Channel } \\
\hline $\mathbf{1}$ & Marketing Cost & $I^{*}$ & \\
\hline & Producer & & 124.10 \\
\hline & Commission agent/ Wholesaler & 75.26 \\
\hline & Retailer & 173.16 & 202.67 \\
\hline & Exporters & 161.14 & - \\
\hline & Total Marketing Cost & - & 538.48 \\
\hline $\mathbf{2}$ & Marketing Margin & 409.56 & 865.25 \\
\hline & Producer & - & - \\
\hline & Commission agent/ Wholesaler & 107.24 & 82.73 \\
\hline & Retailers & 102.83 & - \\
\hline & Exporters & - & 106.35 \\
\hline $\mathbf{3}$ & Total Marketing Margin & 210.07 & 189.08 \\
\hline $\mathbf{4}$ & Total cost + Total Margin & 619.63 & 1054.33 \\
\hline $\mathbf{5}$ & Producer's sale price & 646.91 & 773.33 \\
\hline $\mathbf{6}$ & Consumer's /Buyer's rupee & 981.21 & 1514.48 \\
\hline $\mathbf{7}$ & Price spread & 334.30 & 741.15 \\
\hline $\mathbf{8}$ & Producer's share in buyer's rupee $(\%)$ & 65.93 & 51.05 \\
\hline $\mathbf{9}$ & Marketing Efficiency & 1.94 & 1.04 \\
\hline$*$ Channel-I $=$ Producer $\rightarrow$ Commission agent/Wholesaler $\rightarrow$ Retailer $\rightarrow$ Consumer & \\
\hline$* *$ Channel-II = Producer $\rightarrow$ Commission agent/Wholesaler $\rightarrow$ Exporter $\rightarrow$ International Buyer & \\
\hline & & & \\
\hline
\end{tabular}

Thus, the marketing efficiency of onion was found to be better in case of sale in the domestic market through retailer. From the result it is to be noted that the wholesaler's sale price of onion for retailer in domestic market or channel I (Rs. 981.21) and exporter in export market or Channel II (Rs.1514.48) differed significantly and turned out to be higher in export market due to better quality of produce diverted to exporter as against retailer. The same result was generated by Shah (2015).

In conclusion, onion is an important business to many producers and this is an important crop which helps to increase the economic condition of the farmers. Due to urbanization and globalization, there is rise in demand for onion in both domestic and international market. The study indicated that the marketing efficiency of onion was found to be the higher i.e. 1.94 per cent in case of channel I compare to channel II (1.04 per cent). Thus, the marketing efficiency of onion was found to be better in case of sale in the domestic market through retailer. The result showed low producer's share in consumer price. Market intermediaries are accruing higher margin so the major share of consumers' 
rupee is pocketed by the middlemen. Apart from this, due to lack of marketing system farmers are unable to get remunerative price. Sometimes farmers needed cash after threshold the crop and supposed to be forced sale of their produce and get uneconomic minimum market price. Therefore, for profitable transactions a fair and suitable marketing system of onion is needed in the district. Marketing through co-operative and farmer producer organization should be encouraged to increase the producer's share in consumer rupee. Beside this, effort should be also made to boost the export trade of onion by improving quality and quantity terms.

\section{References}

Baba, S.H., Wani, M.H., Wani, S.A. and Shahid Yousuf (2010) Marketed Surplus and Price Spread of Vegetables in Kashmir Valley. Agricultural Economics Research Review, 23, 115127.

Barakade A.J., Lokhande T.N. and Todkari G.U. (2011) Economics of Onion Cultivation and its Marketing Pattern in Satara district of Maharashtra.
International Journal of Agriculture Sciences, 3(3), 110-117.

Gaurav Joshi (2011) Studied the Analysis of Marketed Surplus and Price Spread of Brinjal in Western Uttar Pradesh. Asian Journal of Management Research, 2(1), 484-490.

Jagtap, M.D. (2014) Price Spread in Marketing of Grapes in Pune District of Maharashtra. International Research Journal of Agricultural Economics and Statistics, 5(2), 176-178.

Shah D. (2015) Relationship between Wholesale Prices, Retail Prices, Export Prices (FOB), Price Realized by Farmers and Details of Contributing Factors for the Price Difference for Onion and Grapes for Maharashtra. Agro-Economic Research CentreReport, Gokhale Institute of Politics and Economics, Pune.

Sashimatsung (2015) Production and Marketing System of Orange in Wokha district of Nagaland: An empirical analysis. International Journal of Development Research, 5(3), 36933697.

\section{How to cite this article:}

Kumud Shukla, Ghanshyam Kumar Pandey, M. Vinaya Kumari, Avinash Vanam and Nahar Singh. 2019. A Study on Marketing Pattern of Onion in Nashik District of Maharashtra, India. Int.J.Curr.Microbiol.App.Sci. 8(04): 151-160. doi: https://doi.org/10.20546/ijcmas.2019.804.016 